\title{
Differentiating symptoms of depression from diabetes-specific distress: relationships with self-care in type 2 diabetes
}

\author{
J. S. Gonzalez • L. M. Delahanty • S. A. Safren • \\ J. B. Meigs $\cdot$ R. W. Grant
}

Received: 3 March 2008 / Accepted: 8 July 2008 / Published online: 9 August 2008

(C) Springer-Verlag 2008

\begin{abstract}
Aims/hypothesis The aim of this study was to examine the relationship between depressive symptoms and diabetesspecific distress and the independent relationships of each of these factors with diabetes self-care. We expected that symptoms of depression would be associated with poorer diabetes self-care, independent of diabetes-specific distress. Methods We surveyed 848 primary care patients with type 2 diabetes using the Harvard Department of Psychiatry/ National Depression Screening Day Scale (HANDS), Problem Areas in Diabetes scale (PAID), Summary of Diabetes Self-Care Activities, and self-reported medication adherence. Results The PAID and HANDS scores were positively correlated in the overall sample $(r=0.54, p<0.0001)$, among those who did not meet diagnostic criteria for major depressive disorder (MDD) based on the HANDS screening result $(n=685 ; r=0.36, p<0.001)$ and in patients who did meet the screening criteria for MDD $(n=163 ; r=0.36$, $p<0.001)$. Higher PAID scores significantly predicted lower levels of diet, exercise and medication adherence (all $p$ values $<0.05$ ). However, once depression symptom scores
\end{abstract}

J. S. Gonzalez $(\bowtie) \cdot$ S. A. Safren

Behavioral Medicine, Department of Psychiatry,

Massachusetts General Hospital and Harvard Medical School,

One Bowdoin Square, 7th Floor,

Boston, MA 02114, USA

e-mail: jsgonzalez@partners.org

L. M. Delahanty

Massachusetts General Hospital Diabetes Center and

Harvard Medical School,

Boston, MA, USA

J. B. Meigs $\cdot$ R. W. Grant

General Medicine Division, Department of Medicine,

Massachusetts General Hospital and Harvard Medical School,

Boston, MA, USA were entered into these models, most relationships were reduced to non-significance, while the HANDS score retained significant relationships with most indices of diabetes selfcare. The same pattern of results was found in the subset of patients who did not screen positive for MDD.

Conclusions/interpretation These results suggest that specific symptoms of depression have a greater negative relationship with diabetes self-care than diabetes-specific distress, even among those patients who do not meet screening criteria for MDD. Interventions that focus on improving the management of specific symptoms of depression may be more effective in improving self-care than those that focus on reducing distress.

Keywords Adherence · Depression .

Diabetes-specific distress · Self-care · Self-management .

Type 2 diabetes
Abbreviations
CES-D
Center for Epidemiological Studies
Depression Scale
DPN diabetic peripheral neuropathy
HANDS
PAID, MDD Harvard Department of Psychiatry/National Depression Screening Day Scale
SDSCA major depressive
in Diabetes scale Summary of Diabetes Self-Care Activities

\section{Introduction}

Measurement inconsistencies complicate the conceptualisation of depression in diabetes and call into question how depression, especially as measured by depressive symptom scales, differs from diabetes-specific distress [1, 2]. We 
previously reported that the relationship between symptoms of depression and worse diabetes self-care is linear and extends to patients who do not meet screening criteria for major depressive disorder (MDD) [3]. We have also observed that higher diabetes-specific distress is associated with poorer self-care [4]. The aim of the present study was to examine the relationship between symptoms of MDD and diabetes-specific distress and the independent relationships of these variables to self-care. Data on the relationship between scores on a measure of diagnostic symptoms of MDD and diabetes-specific distress have not previously been available, and the relationship of the Problem Areas in Diabetes scale (PAID) scores to type 2 diabetes self-care, independent of MDD symptoms, has not been examined.

\section{Methods}

We surveyed primary care patients with type 1 or 2 diabetes. Details of recruitment procedures and characteristics of this sample have been previously reported [3, 4]. Briefly, a total of 1,648 potential participants were contacted and $953(72.4 \%)$ provided informed consent and completed the study survey. The Partners Healthcare System/Massachusetts General Hospital Institutional Review Board approved the study. We excluded patients with type 1 diabetes for this analysis, leaving 909 patients with type 2 diabetes. Missing data further reduced this number for the analyses presented below. Demographic and clinical information for the sample that we were able to compute valid values for our primary independent variables is presented in Table 1.

Total scores from the ten-item Harvard Department of Psychiatry//National Depression Screening Day Scale (HANDS) measured severity of depressive symptoms. The HANDS screens for MDD by assessing symptoms over the prior 2 weeks [5]. The Summary of Diabetes Self-Care Activities (SDSCA) questionnaire measured diabetes selfmanagement over the previous seven days using four subscales: general diet, exercise, self-monitoring of blood glucose and foot care. The specific diet subscale, which includes fruit and vegetable consumption, high-fat food consumption and spacing of carbohydrates, was examined at the item level because of poor inter-item correlations [6]. To assess non-adherence to medication, we asked patients, 'In the past 7 days, on how many days did you miss taking any one of your prescribed medicines?' We dichotomised responses into 'any missed doses' and 'no missed doses'. The Problem Areas in Diabetes (PAID) scale measured total diabetes-specific distress, with 20 items rated on a six-point scale from 'not a problem' to 'a serious problem' [7]. Demographic, diabetes treatment data and Charlson comorbidity scores were derived from survey responses and medical record review $[3,4]$. Because the HANDS and PAID each had high internal reliability $(0.85$ and 0.95 , respectively), we took the mean of available items if no more than $10 \%$ were missing. No other missing data was imputed. We computed $z$ score transformations of both PAID and HANDS scores, where the mean of each scale was zero and each unit represented a standard deviation, and used these variables in the analyses presented below.

We conducted three sets of regression analyses predicting the SDSCA subscale scores: in the first set, the PAID score for the total sample was entered alone; in the second set, the PAID and HANDS scores were entered together for the total sample, and in the third set, the PAID and HANDS scores were entered together, limiting the analysis to those patients who did not screen positive for MDD (i.e. HANDS score $<9$ ). Models predicting the scores for the SDSCA subscales used multiple linear regression and those predicting medication adherence used logistic regression. Analyses were conducted using the SPSS package, version 15.0 (SPSS, Chicago, IL, USA), and all models controlled for

Table 1 Sample characteristics

\begin{tabular}{|c|c|}
\hline Demographics and health variables $(n=848)$ & Value \\
\hline HANDS total score & $5.11(5.07)$ \\
\hline PAID total score & $19.06(20.37)$ \\
\hline \multicolumn{2}{|l|}{ Sex } \\
\hline Female & 47.6 \\
\hline Male & 52.4 \\
\hline \multicolumn{2}{|l|}{ Race and ethnicity } \\
\hline White & 83.1 \\
\hline Black & 8.6 \\
\hline Hispanic & 2.9 \\
\hline Asian & 1.2 \\
\hline Other & 1.3 \\
\hline Not recorded & 2.8 \\
\hline \multicolumn{2}{|l|}{ Marital status } \\
\hline Married or lives with partner & 53.9 \\
\hline Lives alone & 46.1 \\
\hline \multicolumn{2}{|l|}{ Clinic site } \\
\hline Hospital-based & 61.8 \\
\hline Community clinic & 38.2 \\
\hline \multicolumn{2}{|l|}{ Education } \\
\hline Less than high school diploma & 21.9 \\
\hline High school diploma, its equivalent or some college & 55.0 \\
\hline 4 years of college or advanced degree & 23.1 \\
\hline Age (years) & $66.21(12.42)$ \\
\hline Diabetes duration (years) & $9.49(7.55)$ \\
\hline Charlson comorbidity score & $3.06(1.81)$ \\
\hline Total number of medications & $4.29(4.93)$ \\
\hline BMI $\left(\mathrm{kg} / \mathrm{m}^{2}\right)$ & $31.35(6.77)$ \\
\hline \multicolumn{2}{|l|}{ Prescribed insulin } \\
\hline Yes & 26.4 \\
\hline No & 73.6 \\
\hline
\end{tabular}

Values are presented as percentages or mean (SD) 
the same set of covariates as in our previous report to avoid potential demographic and illness-related confounding of measures of depression/diabetes-specific distress [3].

\section{Results}

The PAID and HANDS were completed by 848 respondents and scores were positively correlated $(r=0.54$, $p<0.001)$ in the overall sample, in those patients who did not meet HANDS criteria for MDD $(n=685 ; r=0.36$, $p<0.001)$ and among those who did meet screening criteria for MDD $(n=163 ; r=0.36, p<0.001)$. Table 2 shows that while higher PAID scores were significantly associated with poorer adherence to general dietary recommendations, less spacing of carbohydrates, less frequent exercise, and nonadherence to prescribed medication (when each subscale was examined individually), these relationships were reduced to non-significance when HANDS score was added to the model. In these models, higher levels of depressive symptoms remained significantly associated with each of the diabetes self-care outcomes, independent of PAID score. Higher PAID scores were significantly related to more high-fat food consumption; HANDS score, which was unrelated to high-fat food consumption, did not reduce this relationship. Neither HANDS nor PAID scores were associated with foot care. These relationships were paralleled in the subsample of patients who did not meet screening criteria for MDD, except that HANDS score was negatively related to high-fat food consumption (Table 2). Controlling for prescription of antidepressants did not alter these results (data not shown).

\section{Discussion}

This report responds to a lack of data on the relationship between scores on a diagnostic symptom-based MDD screen and diabetes-specific distress. Our findings suggest that while symptoms of MDD and diabetes-specific distress are related, sharing $29 \%$ of their variance in the overall sample and $13 \%$ among those who did not meet screening criteria for MDD, they are independent constructs. Our results also show that symptoms of depression are more

Table 2 Diabetes-specific distress vs depressive symptomology predicting diabetes self-care

\begin{tabular}{|c|c|c|c|c|c|c|c|c|}
\hline \multirow[t]{2}{*}{ SDSCA subscale } & \multicolumn{2}{|c|}{ PAID in overall sample ${ }^{a}$} & \multicolumn{3}{|c|}{ PAID and HANDS in overall sample ${ }^{a}$} & \multicolumn{3}{|c|}{$\begin{array}{l}\text { PAID and HANDS in those not meeting } \\
\text { screening criteria for } \text { MDD }^{b}\end{array}$} \\
\hline & $\begin{array}{l}B(\mathrm{SE}) \text { or } \\
\text { OR }(95 \% \mathrm{CI})^{\mathrm{c}}\end{array}$ & $p$ value & $\begin{array}{l}B(\mathrm{SE}) \text { or } \\
\text { OR }(95 \%\end{array}$ & & $p$ value & $\begin{array}{l}B(\mathrm{SE}) \text { or } \\
\text { OR }(95 \%\end{array}$ & & $p$ value \\
\hline \multirow[t]{2}{*}{ General diet } & $-0.33(0.09)$ & $<0.001$ & PAID & $-0.11(0.10)$ & 0.259 & PAID & $-0.24(0.12)$ & 0.055 \\
\hline & & & HANDS & $-0.44(0.10)$ & $<0.001$ & HANDS & $-0.67(0.20)$ & 0.001 \\
\hline \multirow[t]{2}{*}{ Fruits and vegetables ${ }^{\mathrm{d}}$} & $-0.15(0.10)$ & 0.133 & PAID & $0.04(0.12)$ & 0.704 & PAID & $0.02(0.14)$ & 0.879 \\
\hline & & & HANDS & $-0.40(0.12)$ & 0.001 & HANDS & $-0.86(0.24)$ & $<0.001$ \\
\hline \multirow[t]{2}{*}{ Carbohydrate spacing } & $-0.23(0.10)$ & 0.029 & PAID & $0.05(0.12)$ & 0.646 & PAID & $-0.02(0.15)$ & 0.892 \\
\hline & & & HANDS & $-0.56(0.12)$ & $<0.001$ & HANDS & $-0.78(0.25)$ & 0.002 \\
\hline \multirow[t]{2}{*}{ High-fat foods } & $0.20(0.07)$ & 0.007 & PAID & $0.25(0.08)$ & 0.003 & PAID & $0.32(0.10)$ & 0.002 \\
\hline & & & HANDS & $-0.10(0.08)$ & 0.197 & HANDS & $-0.35(0.16)$ & 0.033 \\
\hline \multirow[t]{2}{*}{ Exercise } & $-0.18(0.09)$ & 0.042 & PAID & $0.03(0.10)$ & 0.777 & PAID & $-0.11(0.13)$ & 0.379 \\
\hline & & & HANDS & $-0.42(0.10)$ & $<0.001$ & HANDS & $-0.51(0.21)$ & 0.015 \\
\hline \multirow[t]{2}{*}{ Glucose monitoring } & $0.05(0.10)$ & 0.646 & PAID & $0.18(0.12)$ & 0.125 & PAID & $0.16(0.15)$ & 0.270 \\
\hline & & & HANDS & $-0.27(0.12)$ & 0.021 & HANDS & $0.17(0.24)$ & 0.486 \\
\hline \multirow[t]{2}{*}{ Foot care ${ }^{\mathrm{d}}$} & $-0.05(0.10)$ & 0.599 & PAID & $0.02(0.12)$ & 0.877 & PAID & $-0.09(0.14)$ & 0.548 \\
\hline & & & HANDS & $-0.14(0.11)$ & 0.212 & HANDS & $-0.21(0.23)$ & 0.374 \\
\hline \multirow{2}{*}{$\begin{array}{l}\text { Medication } \\
\text { non-adherence }\end{array}$} & $1.30(1.08-1.56)$ & 0.006 & PAID & $1.00(0.80-1.25)$ & 0.987 & PAID & $1.14(0.86-1.51)$ & 0.363 \\
\hline & & & HANDS & $1.63(1.32-2.02)$ & $<0.001$ & HANDS & $1.63(1.02-2.60)$ & 0.041 \\
\hline
\end{tabular}

All models are adjusted for clinic site, sex, education, age, marital status, total medications, BMI, Charlson comorbidity score and prescription of insulin. The PAID was used to assess diabetes-specific stress; the HANDS was used to assess depressive symptoms

${ }^{a}$ The number of subjects for these analyses ranges from 773 to 816

${ }^{\mathrm{b}}$ Analysis limited to those participants who scored $<9$ on the HANDS, which indicates that the presence of a major depressive episode is unlikely. The number of participants for these analyses ranges from 628 to 661

${ }^{\mathrm{c}}$ Data are unstandardised regression coefficients $(B)$ from multiple regression in all models, except for medication non-adherence, where data are ORs with $95 \%$ CIs calculated by logistic regression. In these models, non-adherence is coded as 1 and adherence is coded as 0 . OR reflects the increase in odds of non-adherence associated with a one point increase on either the PAID or HANDS. $B$ reflects the change in the dependent variable associated with a one-unit change in the independent variable. Because analyses used standardised values of PAID and HANDS, a one unit change in the independent variable is an SD. SE is the standard error of $B$

${ }^{\mathrm{d}}$ These models contained race (white vs other) as an additional covariate 
consistently related to worse diabetes self-care than diabetes-specific distress.

Previously, Fisher and colleagues reported that diabetesspecific distress was a better predictor of diabetes self-care than symptoms of depression or a diagnosis of MDD [1]. Our analysis demonstrates that depressive symptoms are a better predictor of diabetes self-care than diabetes-specific distress, even among those unlikely to meet criteria for MDD. These findings may not be contradictory. First, it is possible that when Fisher and colleagues controlled for MDD, the remaining variance in scores on the Center for Epidemiological Studies Depression Scale (CES-D) was more difficult to differentiate from diabetes-specific distress. Second, our measure of depression focused on symptoms of MDD according to the Diagnostic and Statistical Manual of Mental Disorders, 4th edition (DSM-IV), in contrast with the item content of the CES-D, which also captures general distress and is less specific to symptoms of MDD.

Our study is limited by our use of self-reports for self-care, which may overestimate true levels. Further, these crosssectional data, from a relatively ethnically homogeneous sample, limit causal inferences. However, we have recently published longitudinal data that support the directionality of this relationship and show that baseline symptoms of depression and increases in depressive symptoms over time are each significantly associated with worse diabetes self-care 9 months later, even when baseline levels of self-care are controlled [8]. Thus, despite their correlation, our data suggest that depressive symptoms may be more problematic for diabetes self-care than the more general emotional reactions to diabetes captured by the PAID, even among those unlikely to meet criteria for MDD. However, our analysis does not imply that these depressive symptoms are unrelated to diabetes. Our measure of depressive symptoms did not ask patients about causes of these symptoms. It may very well be that these depressive symptoms are related to the experience of the illness for many patients. For example, we have previously reported that, according to our data, the number of prescribed medications, number of comorbid conditions and treatment with insulin are each significantly associated with depressive symptoms [3]. Furthermore, a detailed and theoretically driven analysis of factors related to depression in patients with diabetic peripheral neuropathy (DPN) showed that symptoms of depression are related to objective indicators of the severity of DPN, DPN-symptoms, beliefs about DPN, restrictions in activities, and changes in social roles because of DPN [9]. Thus, symptoms of depressed mood, diminished interest and pleasure, worthlessness and self-blame, concentration difficulties, appetite disruption, sleep disturbance, fatigue, hopelessness and suicidal ideation could be influenced by the patient's experience with diabetes, and these symptoms in turn could complicate the patient's ability to successfully manage self-care. Given our findings showing that these depressive symptoms, even at subclinical levels, are more closely related to diabetes self-care than diabetes-specific distress, more research is needed to evaluate whether interventions specifically aimed at improving the recognition and management of these symptoms may have positive effects on self-care. As the effect sizes for these relationships are in the small to medium range, such interventions may be most effective in improving diabetes self-care and disease outcomes if they are wide-reaching and/ or directly target self-care.

Acknowledgements J. S. Gonzalez's and S. A. Safren's efforts on this manuscript were supported by a grant from the National Institute of Mental Health (no. 1R01MH078571). J. B. Meigs was supported by an American Diabetes Association Career Development Award and by National Institute of Diabetes and Digestive and Kidney Diseases (NIDDK) grant no. K24 DK080140. J. B. Meigs currently has research grants from GlaxoSmithKline and sanofi-aventis, and consults for GlaxoSmithKline, sanofi-aventis, Interleukin Genetics, Kalypsis and Outcomes Science. R. W. Grant is supported by a Career Development Award (no. K23 DK067452) from the NIDDK.

Duality of interest This study was supported by a grant from Pfizer, with additional support from sanofi-aventis and the Massachusetts General Hospital Clinical Research Program.

\section{References}

1. Fisher L, Skaff MM, Mullan JT et al (2007) Clinical depression versus distress among patients with type 2 diabetes: not just a question of semantics. Diabetes Care 30:542-548

2. Hermanns N, Kulzer B, Krichbaum M, Kubiak T, Haak T (2006) How to screen for depression and emotional problems in patients with diabetes: comparison of screening characteristics of depression questionnaires, measurement of diabetes-specific emotional problems and standard clinical assessment. Diabetologia 49:469-477

3. Gonzalez JS, Safren SA, Cagliero E et al (2007) Depression, selfcare, and medication adherence in type 2 diabetes: relationships across the full range of symptom severity. Diabetes Care 30:22222227

4. Delahanty LM, Grant RW, Wittenberg E et al (2007) Association of diabetes-related emotional distress with diabetes treatment in primary care patients with type 2 diabetes. Diabet Med 24:48-54

5. Baer L, Jacobs DG, Meszler-Reizes J et al (2000) Development of a brief screening instrument: The HANDS. Psychother Psychosom 69:35-41

6. Toobert DJ, Hampson SE, Glasgow RE (2000) The summary of diabetes self-care activities measure: results from 7 studies and a revised scale. Diabetes Care 23:943-950

7. Polonsky WH, Anderson BJ, Lohrer PA et al (1995) Assessment of diabetes-related distress. Diabetes Care 18:754-760

8. Gonzalez JS, Safren SA, Delahanty LM, et al. Symptoms of depression prospectively predict poorer self-care in patients with type 2 diabetes. Diabetic Medicine doi:10.1111/j.1464-5491.2008.02535.x

9. Vileikyte L, Leventhal H, Gonzalez JS et al (2005) Diabetic peripheral neuropathy and depression: the association revisited. Diabetes Care 28:2378-2383 